\title{
Investigation of the Role of Dinutuximab Beta-Based Immunotherapy in the SIOPEN High-Risk Neuroblastoma 1 Trial (HR-NBL1)
}

\author{
Ruth Ladenstein ${ }^{1, *,+}$, Ulrike Pötschger ${ }^{2,+}{ }^{+}$, Dominique Valteau-Couanet ${ }^{3}$, Roberto Luksch ${ }^{4}$, \\ Victoria Castel ${ }^{5}{ }^{\circ}$, Shifra Ash ${ }^{6}$, Geneviève Laureys ${ }^{7}{ }^{(0)}$, Penelope Brock ${ }^{8}$, Jean Marie Michon ${ }^{9}$, \\ Cormac Owens ${ }^{10}$, Toby Trahair ${ }^{11}$, Godfrey Chi Fung Chan ${ }^{12}$, Ellen Ruud ${ }^{13}$, Henrik Schroeder ${ }^{14}$, \\ Maja Beck-Popovic ${ }^{15}$, Guenter Schreier ${ }^{16}{ }^{1}$, Hans Loibner ${ }^{17}$, Peter Ambros ${ }^{2}$, Keith Holmes ${ }^{18}$, \\ Maria Rita Castellani ${ }^{4}$, Mark N. Gaze ${ }^{19}\left(\mathbb{D}\right.$, Alberto Garaventa ${ }^{20}$, Andrew D.J. Pearson ${ }^{21}$ and \\ Holger N. Lode 22 (D)
}

1 St. Anna Children's Hospital and Children's Cancer Research Institute (CCRI), Department of Paediatrics, Medical University, 1090 Vienna, Austria

2 Children's Cancer Research Institute (CCRI), St. Anna Kinderkrebsforschung, Department of Paediatrics, Medical University, 1090 Vienna, Austria; ulrike.poetschger@ccri.at (U.P.); peter.ambros@ccri.at (P.A.)

3 Children and Adolescent Oncology Department, Gustave Roussy, Paris-Sud, University, 94805 Villejuif, France; Dominique.VALTEAU@gustaveroussy.fr

4 Fondazione IRCCS Istituto Nazionale dei Tumori, 20133 Milan, Italy; roberto.luksch@istitutotumori.mi.it (R.L.); rita.castellani@istitutotumori.mi.it (M.R.C.)

5 Hospital Universitario y Politecnico La Fe, 46026 Valencia, Spain; castel_vic@gva.es

6 Schneider Children's Medical Center of Israel, Sackler Faculty of Medicine Tel Aviv University, Petach, Tikvah 49202, Israel; shifraa@clalit.org.il

7 University Hospital Ghent, 9000 Ghent, Belgium; genevieve.laureys@ugent.be

8 Great Ormond Street Hospitalfor Children, London WC1N 3JH, UK; peppybrock@gmail.com

9 Institut Curie, 75248 Paris, France; jean.michon@curie.fr

10 Paediatric Haematology/Oncology, Our Lady's Children's Hospital, Crumlin, D12 N512 Dublin, Ireland; cormac.owens@olchc.ie

11 Sydney Children's Hospital, Randwick NSW 2031, Australia;

Toby.Trahair@SESIAHS.HEALTH.NSW.GOV.AU

12 Department of Paediatrics \& Adolescent Medicine, Queen Mary Hospital, Hong Kong, China; gcfchan@hku.hk

13 Rikshospitalet, 0027 Oslo, Norway; ellen.ruud@rikshospitalet.no

14 Department of Paediatrics, University Hospital of Aarhus, 8200 Aarhus, Denmark; henrik.schroeder@skejby.rm.dk

15 Department of Paediatrics, University Hospital Lausanne, 1011 Lausanne, Switzerland; Maja.Beck-Popovic@chuv.ch

16 AIT Austrian Institute of Technology GmbH, 8020 Graz, Austria; guenter.schreier@ait.ac.at

17 Apeiron Biologics AG, 1030 Vienna, Austria; hans.loibner@hl-bioscience.com

18 St George's Hospital, Department Paediatric Surgery, London SW17 0QT, UK; kholmes@doctors.org.uk

19 National Institute for Health Research University College London Hospitals Biomedical Research Centre, University College London Hospitals NHS Foundation Trust, Department of Oncology, London W1 2PG, UK; mark.gaze@uclh.nhs.uk

20 IRCCS Istituto Giannina Gaslini, 16148 Genova, Italy; albertogaraventa@ospedale-gaslini.ge.it

21 Institute of Cancer Research, Royal Marsden Hospital, Sutton SM5 2NG, UK; andy1pearson@btinternet.com

22 Department of Pediatric Hematology and Oncology, University Medicine Greifswald, 17489 Greifswald, Germany; holger.lode@uni-greifswald.de

* Correspondence: ruth.ladenstein@ccri.at; Tel.: +43(1)-40470-4750; Fax: +43(1)-40470-7430

+ The authors contributed equally to this work and share first authorship. 


\begin{abstract}
To explore the effects of immunotherapy in the International Society of Paediatric Oncology Europe Neuroblastoma Group SIOPEN high-risk neuroblastoma 1 trial (HR-NBL1 trial), two cohorts were studied: one prior to and one after the introduction of dinutuximab beta. All patients received standard induction and high-dose therapy (HDT) with autologous stem cell rescue (ASCR); the local control comprised surgery and radiotherapy to the primary tumour site, followed by isotretinoin. A landmark timepoint of 109 days, resulting from the median time between ASCR and initiation of immunotherapy, was used to define patients' eligibility in the pre-immunotherapy analysis cohort. Median follow-up was 5.8 years (inter-quartile range (IQR): 4.2-8.2 years) for 844 eligible patients balanced for risk factors, such as age, sex, stage $4, M Y C N$ amplification and response prior to HDT. The five-year event-free and overall survival (95\% confidence interval (CI) of 466 patients not receiving immunotherapy was $42 \%(38-47 \%)$ and $50 \%(46-55 \%)$ but was $57 \%(51-62 \%)$ and $64 \%(59-69 \%)$ for 378 patients receiving immunotherapy $(p<0.001)$. A multivariate analysis identified absence of immunotherapy ( $p=0.0002$, hazard ratio (HR) 1.573); type of HDT ( $p=0.0029$, HR 1.431); less than complete response prior to maintenance therapy $(p=0.0043$, HR 1.494) and $>1$ metastatic compartment at diagnosis $(p<0.001$, HR 2.665) as risk factors for relapse or progression. Results suggest an important role for dinutuximab beta-based immunotherapy within the treatment concepts applied in HR-NBL1/SIOPEN.
\end{abstract}

Keywords: high-risk neuroblastoma; immunotherapy; dinutuximab beta

\title{
1. Introduction
}

High-risk neuroblastoma defined by metastatic diseases over the age of 12 or 18 months [1] and MYCN amplification at any age remain associated with long-term survival rates of only $40 \%[2,3]$. Treatment approaches comprise intensive induction [4,5], consolidation with high-dose chemotherapy (HDT) and autologous stem cell rescue (ASCR) [3,6], and isotretinoin as maintenance therapy.

As the disialoganglioside $\mathrm{GD}_{2}$ is expressed on the majority of neuroblastoma cells, with minimal expression on normal cells, it is a suitable target for immunotherapy [7]. Therefore, human/mouse chimeric anti-GD $\mathrm{GD}_{2}$ antibody ch14.18, dinutuximab, produced in SP2/0 cells was developed and investigated in clinical trials [8]. In Europe, ch14.18 was re-cloned in Chinese hamster ovarian (CHO) cells (dinutuximab beta) [9] for clinical trials of International Society of Paediatric Oncology Europe Neuroblastoma Group SIOPEN. The tolerability and activity of dinutuximab beta was first evaluated in a dose schedule of $20 \mathrm{mg} / \mathrm{m}^{2}$ given on five consecutive days by an $8 \mathrm{~h}$ infusion [10]. In 2006, SIOPEN opened a randomised trial to compare dinutuximab beta and isotretinoin with isotretinoin alone in patients with high-risk neuroblastoma. However, in 2007, the results of the Children's Oncology Group (COG) ANBL0032 trial were communicated, followed by publication in 2010 [7], demonstrating that two-year event-free survival (EFS) and overall survival (OS) of patients with high-risk neuroblastoma receiving dinutuximab and cytokines (granulocyte-macrophage colony stimulating factor and interleukin-2), in addition to isotretinoin, were significantly higher by $20 \%$ and $11 \%$, respectively [7], compared to those patients receiving isotretinoin alone. Therefore, continuation of the SIOPEN randomised trial was believed to be no longer feasible nor considered ethical, and the study design was modified to allow all patients to receive dinutuximab beta with or without interleukin-2. The altered randomisation opened on 22 October 2009 to investigate the role of subcutaneous interleukin-2 (sc-IL-2) with dinutuximab beta and assigned patients to dinutuximab beta alone or with sc-IL-2 [11]. All patients received oral isotretinoin [12]. The trial showed that the addition of sc-IL-2 to immunotherapy with dinutuximab beta, given as an $8 \mathrm{~h}$ infusion, did not improve outcome but increased toxicity.

In this report, we aim to assess the contribution of dinutuximab beta-based immunotherapy to the outcome of patients with high-risk neuroblastoma in the International Society of Paediatric Oncology 
Europe Neuroblastoma Group High-Risk Neuroblastoma 1 (HR-NBL1/SIOPEN) trial by investigating the survival of patients in sequential eras with the same eligibility criteria treated with (immunotherapy population (IP), 2009-2013) [12] or without immunotherapy (control population (CP), 2002-2009).

\section{Results}

\subsection{Patient Characteristics}

According to the inclusion criteria for the analysis, 844 patients enrolled in 146 SIOPEN member hospitals/institutions in 19 countries were eligible (378 in the IP and 466 in the CP) (Figure 1). Median follow-up was 5.8 years (inter-quartile range (IQR): 4.2 to 8.2 years). The median age of patients at diagnosis was 2.9 years (IQR: 1.8. to 3.8).

\begin{tabular}{|c|}
\hline $2002-2009$ \\
1347 patients
\end{tabular}

\begin{tabular}{|c|}
\hline $2009-20013$ \\
861 patients
\end{tabular}

High-dose chemotherapy

after sufficient response with Rapid Cojec \pm TVD

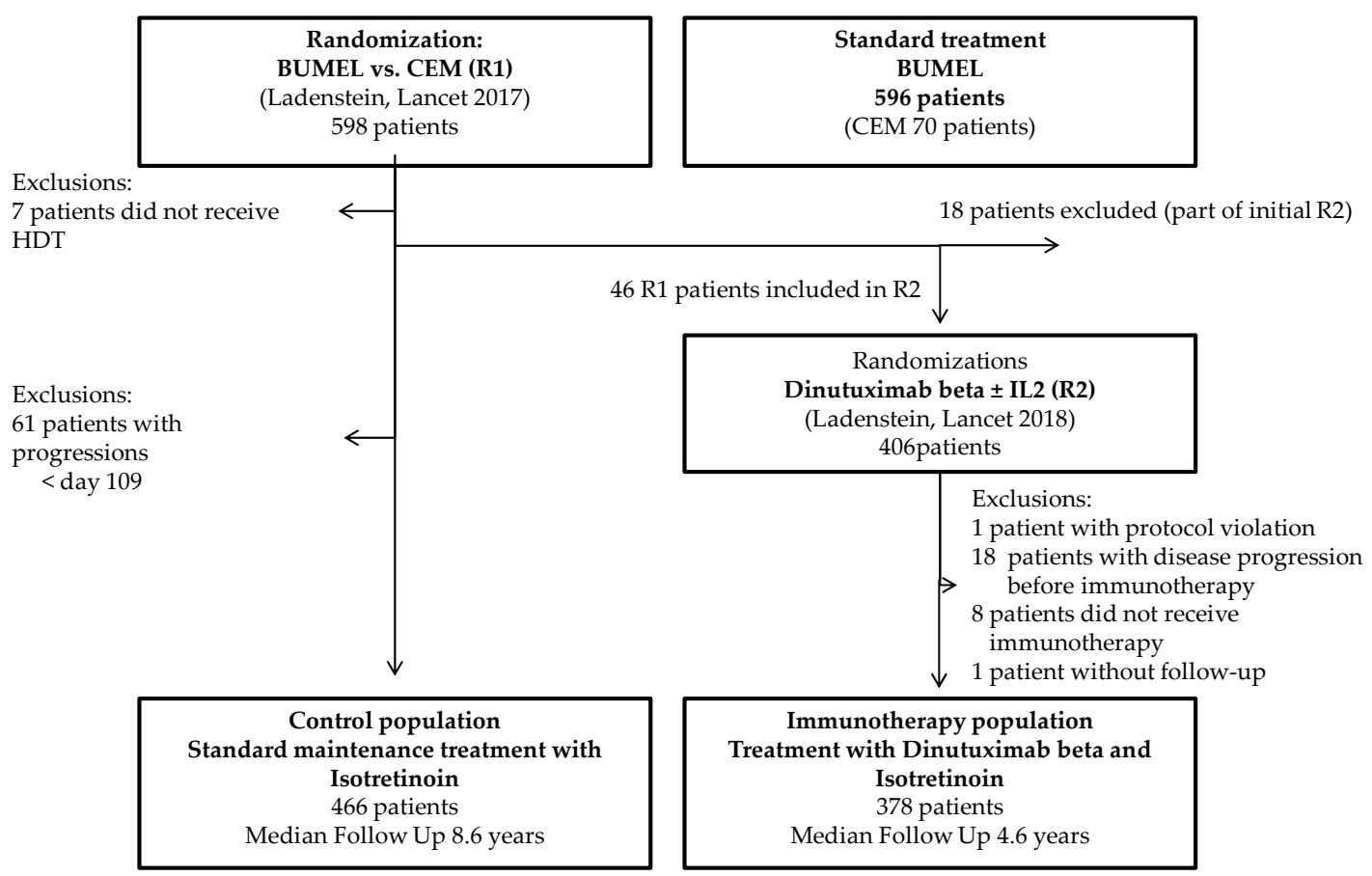

Figure 1. Flow chart for the analysis cohort. HDT (high-dose chemotherapy); BuMel (high-dose chemotherapy with busulfan and melphalan; CEM (high-dose chemotherapy with carboplatin, etoposide and melphalan); R1 (high-dose chemotherapy randomisation); R2 (immunotherapy randomisation) and IL-2 (interleukin-2).

(Table 1).

Both populations were balanced for sex, stage 4, MYCN amplification and response prior to HDT

\subsection{Survival}

The five-year EFS was 57\% (95\% CI: 51-62\%) for IP, compared to $42 \%$ (95\% CI: 38-47\%) for CP patients $(p<0.001)$ (Figure 2A). The five-year overall survival (OS) for the IP was 64\% (95\% CI: 59-69\%), compared to $50 \%$ (95\% CI: $46-55 \%$ ) for CP patients (Figure $2 \mathrm{~B}$ ). 
Table 1. Characteristics of the control and immunotherapy populations. $\mathrm{N}=$ number; $\%=$ percentage; MNA = MYCN amplification; no = not present and yes = present; $\mathrm{MC}=$ metastatic compartments; TVD = topotecan, vincristine and doxorubicin; HDT = high-dose chemotherapy; BuMel = high-dose chemotherapy with busulfan and melphalan; CEM = high-dose chemotherapy with carboplatin, etoposide and melphalan; $\mathrm{NR}=$ not reported; $\mathrm{CR}=$ complete remission; $\mathrm{VGPR}=$ very good partial remission; $\mathrm{PR}=$ partial remission; $\mathrm{CME}=$ complete macroscopic excision and $\mathrm{IME}=$ incomplete macroscopic excision.

\begin{tabular}{|c|c|c|c|c|c|}
\hline \multirow{2}{*}{ Characteristics } & & \multicolumn{2}{|c|}{ Control Population } & \multicolumn{2}{|c|}{$\begin{array}{c}\text { Immunotherapy } \\
\text { Population }\end{array}$} \\
\hline & & $n$ & $\%$ & $n$ & $\%$ \\
\hline Total & number & 466 & & 378 & \\
\hline \multirow[t]{2}{*}{ Sex } & Female & 180 & $39 \%$ & 140 & $37 \%$ \\
\hline & Male & 286 & $61 \%$ & 238 & $63 \%$ \\
\hline \multirow[t]{4}{*}{ Age } & $<1.5$ years & 64 & $13 \%$ & 55 & $14 \%$ \\
\hline & $\begin{array}{l}1.5-<5 \\
\text { years }\end{array}$ & 333 & $71 \%$ & 254 & $67 \%$ \\
\hline & $\geq 5$ years & 69 & $15 \%$ & 69 & $18 \%$ \\
\hline & Median & 2.70 & & 2.87 & \\
\hline \multirow[t]{3}{*}{ Stage } & Localised & 60 & $13 \%$ & 32 & $8 \%$ \\
\hline & Stage 4 & 406 & $87 \%$ & 339 & $90 \%$ \\
\hline & Stage $4 \mathrm{~s}$ & 0 & $0 \%$ & 7 & $2 \%$ \\
\hline \multirow[t]{3}{*}{ MYCN Stage 4} & $M N A N R$ & 27 & $6 \%$ & 16 & $4 \%$ \\
\hline & MNA no & 217 & $57 \%$ & 197 & $61 \%$ \\
\hline & MNA yes & 162 & $43 \%$ & 126 & $39 \%$ \\
\hline \multirow[t]{6}{*}{ MC } & NR & 23 & $5 \%$ & 23 & $6 \%$ \\
\hline & 0 & 60 & $14 \%$ & 32 & $9 \%$ \\
\hline & 1 & 70 & $16 \%$ & 35 & $10 \%$ \\
\hline & 2 & 136 & $31 \%$ & 112 & $32 \%$ \\
\hline & 3 & 120 & $27 \%$ & 112 & $32 \%$ \\
\hline & $>3$ & 57 & $13 \%$ & 64 & $18 \%$ \\
\hline \multirow[t]{3}{*}{ TVD given } & NR & 23 & $5 \%$ & 10 & $3 \%$ \\
\hline & No & 391 & $88 \%$ & 250 & $68 \%$ \\
\hline & Yes & 52 & $12 \%$ & 118 & $32 \%$ \\
\hline \multirow[t]{2}{*}{ Surgery } & CME & 318 & $76 \%$ & 261 & $75 \%$ \\
\hline & IME & 101 & $24 \%$ & 87 & $25 \%$ \\
\hline \multirow[t]{4}{*}{ Status prior HDT } & NR & 25 & $5 \%$ & 33 & $9 \%$ \\
\hline & $\mathrm{CR}$ & 174 & $39 \%$ & 116 & $34 \%$ \\
\hline & VGPR & 159 & $36 \%$ & 149 & $43 \%$ \\
\hline & PR & 108 & $24 \%$ & 80 & $23 \%$ \\
\hline \multirow[t]{2}{*}{ HDT } & BuMel & 257 & $55 \%$ & 348 & $92 \%$ \\
\hline & CEM & 209 & $45 \%$ & 30 & $8 \%$ \\
\hline \multirow[t]{4}{*}{ Status prior Maintenance } & NR & 58 & $12 \%$ & 17 & $4 \%$ \\
\hline & $\mathrm{CR}$ & 258 & $63 \%$ & 210 & $58 \%$ \\
\hline & VGPR & 93 & $23 \%$ & 99 & $27 \%$ \\
\hline & PR & 57 & $14 \%$ & 52 & $14 \%$ \\
\hline
\end{tabular}

The cumulative incidence of relapse/progression (CIR) at five years was $41 \%$ (95\% CI: $37-47 \%$ ) for the IP and 57\% (95\% CI: 53-61\%) for the CP patients ( $p<0.001)$. At the last follow-up, 153 patients of the IP had an event versus 272 of CP patients. The cumulative incidence of non-relapse mortality was $2 \%(95 \%$ CI: $1-4 \%)$ in the IP and $1 \%(95 \% \mathrm{CI}<1-2 \%)$ in the CP (Figure $2 \mathrm{C}$ ). 


\subsection{Influence of Risk Factors}

Disease status prior to maintenance therapy was available in 769/844 (91\%) patients (Table 2). Older age; stage 4; involvement of more than one metastatic compartment (MC); disease status prior to maintenance therapy; addition of topotecan, vincristine and doxorubicin (TVD) and use of carboplatin, etoposide and melphalan (CEM) as HDT were associated with lower EFS (Figure 3) in the population analysed. Patients with lymph nodes as their only MC (five-year EFS 60\% (95\% CI: 36-78\%)) had a similar EFS as patients with other isolated metastatic sites (five-year EFS 60\% (95\% CI: 49-70\%)).

A)

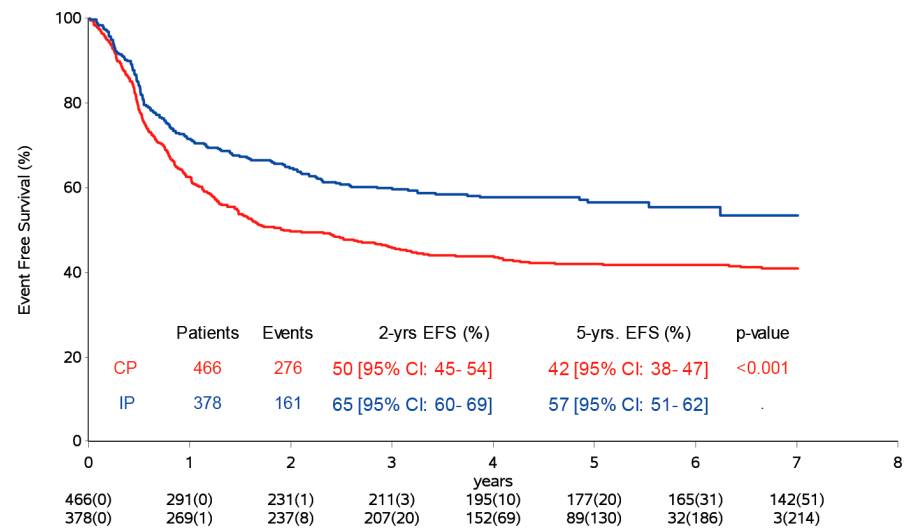

B)

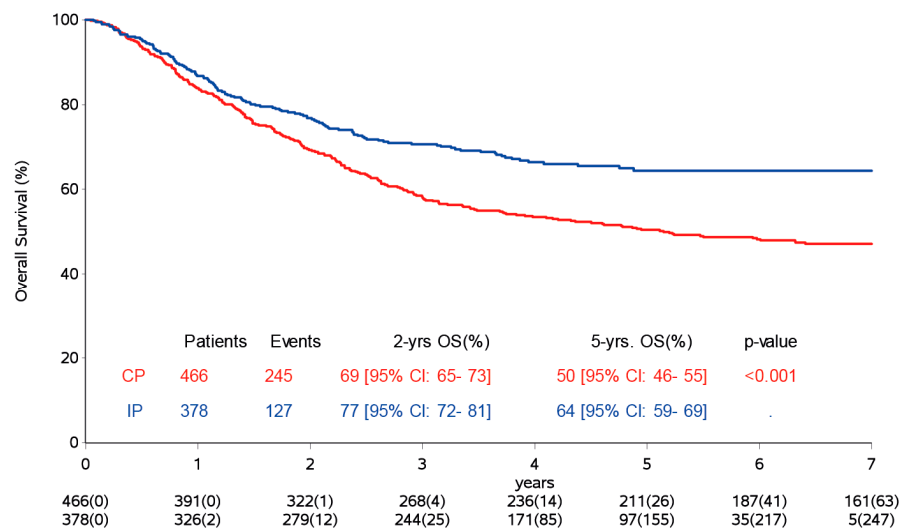

C)

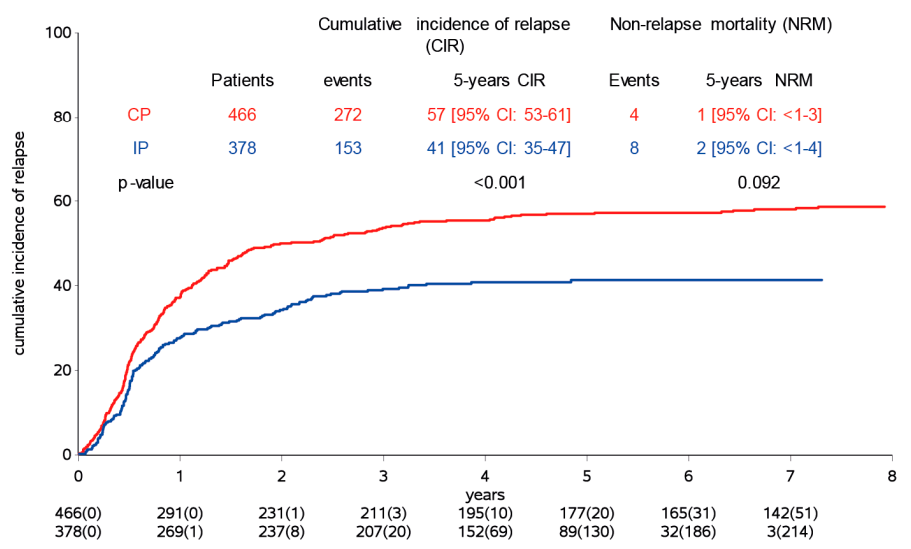

Figure 2. Analysis population comparing control population versus immunotherapy population. (A) Event-free survival, (B) overall survival and (C) cumulative incidence of progression/relapse. CP (control population), IP (immunotherapy population), CIR (cumulative incidence of relapse) and NRM (non-relapse mortality). 
Table 2. Outcomes according to risk factors and subgroups. (2A) Event-free survival and (2B) overall survival. Pts (patients); $95 \% \mathrm{CI}$ (95\% confidence interval); $p$ value (probability value for A: comparison according to risk factor and B: for interaction); MNA (MYCN amplification); - (not present) and + (present); MC (metastatic compartments); TVD (topotecan, vincristine and doxorubicin); HDT (high-dose chemotherapy); BuMel (high-dose chemotherapy with busulfan and melphalan); CEM (high-dose chemotherapy with carboplatin, etoposide and melphalan); CR (complete remission); VGPR (very good partial remission; PR (partial remission); CME (complete macroscopic excision) and IME (incomplete macroscopic excision).

\begin{tabular}{|c|c|c|c|c|c|c|c|c|c|}
\hline \multicolumn{2}{|r|}{ Characteristics } & \multicolumn{3}{|c|}{ Total Population } & \multicolumn{2}{|c|}{ Control Group } & \multicolumn{2}{|c|}{ Immunotherapy Group } & \multirow[t]{2}{*}{$p$-Value ${ }^{\mathrm{B}}$} \\
\hline (A) & Event Free Survival & Events/Pts & 5-years EFS (95\% CI) & $p$-value ${ }^{\mathrm{A}}$ & Events/Pts & 5-years EFS $(95 \% \mathrm{CI})$ & Events/Pts & 5-years EFS (95\% CI) & \\
\hline Total & & 844 Pts & & & 466 Pts & & 378 Pts & & \\
\hline \multirow[t]{2}{*}{ Sex } & female & $164 / 320$ & $49(44-55)$ & 0.803 & $105 / 180$ & $43(36-50)$ & $59 / 140$ & $57(49-65)$ & 0.938 \\
\hline & male & $273 / 524$ & $48(44-52)$ & & $171 / 286$ & $41(36-47)$ & $102 / 238$ & $56(49-62)$ & \\
\hline \multirow[t]{3}{*}{ Age } & $<1.5$ years & $48 / 119$ & $60(51-68)$ & 0.007 & $27 / 64$ & $59(46-70)$ & $21 / 55$ & $61(47-73)$ & 0.161 \\
\hline & $1.5-<5$ years & $302 / 587$ & $49(44-53)$ & & $194 / 333$ & $42(37-47)$ & $108 / 254$ & $57(50-63)$ & \\
\hline & $\geq 5$ years & $87 / 138$ & $38(30-47)$ & & $55 / 69$ & $25(16-36)$ & $32 / 69$ & $53(40-64)$ & \\
\hline \multirow{4}{*}{ Stage } & Stage 4 & $410 / 745$ & $45(42-49)$ & & $262 / 406$ & $37(32-42)$ & $148 / 339$ & $56(50-61)$ & \\
\hline & Stage $4 \mathrm{~s}$ & $2 / 7$ & $71(26-92)$ & & - & - & $2 / 7$ & $71(26-92)$ & \\
\hline & Stage 4 MNA no- & $236 / 414$ & $43(38-48)$ & 0.819 & $147 / 217$ & $33(27-39)$ & $89 / 197$ & $54(47-61)$ & 0.25 \\
\hline & Stage 4 MNA yes+ & $154 / 288$ & $47(41-53)$ & & $98 / 162$ & $42(34-49)$ & $56 / 126$ & $55(45-63)$ & \\
\hline \multirow[t]{4}{*}{ MC } & 0 & $25 / 92$ & $72(62-80)$ & $<0.001$ & $14 / 60$ & $67(63-85)$ & $11 / 32$ & $66(47-79)$ & 0.025 \\
\hline & 1 & $43 / 105$ & $60(50-69)$ & & $34 / 70$ & $54(42-65)$ & $9 / 35$ & $71(50-85)$ & \\
\hline & 2 & $136 / 248$ & $46(39-52)$ & & $85 / 136$ & $39(31-47)$ & $51 / 112$ & $55(45-63)$ & \\
\hline & $>3$ & $76 / 121$ & $37(28-46)$ & & $42 / 57$ & $28(17-40)$ & $34 / 64$ & $45(32-57)$ & \\
\hline \multirow[t]{2}{*}{ TVD } & no & $322 / 641$ & $50(46-54)$ & 0.024 & $225 / 391$ & $44(39-49)$ & $97 / 250$ & $61(54-67)$ & 0.732 \\
\hline & yes & $100 / 170$ & $39(32-47)$ & & $38 / 52$ & $27(16-39)$ & $62 / 118$ & 45 (34-55) & \\
\hline \multirow[t]{2}{*}{ Surgery } & CME & $288 / 578$ & $50(46-54)$ & 0.123 & $183 / 318$ & $43(38-49)$ & $105 / 260$ & $59(52-65)$ & 0.946 \\
\hline & IME & $106 / 188$ & $45(38-52)$ & & $66 / 101$ & $38(29-48)$ & $40 / 87$ & $53(42-63)$ & \\
\hline \multicolumn{10}{|c|}{ Status Prior HDT } \\
\hline & CR & $134 / 290$ & $54(48-59)$ & 0.022 & $86 / 174$ & $51(43-58)$ & $48 / 116$ & $57(47-66)$ & 0.294 \\
\hline
\end{tabular}


Table 2. Cont.

\begin{tabular}{|c|c|c|c|c|c|c|c|c|c|c|}
\hline \multicolumn{3}{|c|}{ VGPR } & $171 / 308$ & $45(39-51)$ & & $104 / 159$ & $36(29-44)$ & $67 / 149$ & $55(46-63)$ & \\
\hline \multicolumn{3}{|c|}{ PR } & $104 / 188$ & $45(38-53)$ & & $70 / 108$ & $38(29-47)$ & $34 / 80$ & $57(45-67)$ & \\
\hline \multicolumn{3}{|c|}{ Characteristics } & \multicolumn{3}{|c|}{ Total Population } & \multicolumn{2}{|c|}{ Control Group } & \multicolumn{2}{|c|}{ Immunotherapy Group } & $p$-value ${ }^{\mathrm{B}}$ \\
\hline (A) & Even & Irvival & Events/Pts & 5-years EFS (95\% CI) & $p$-value ${ }^{A}$ & Events/Pts & 5-years EFS (95\% CI) & Events/Pts & 5-years EFS (95\% CI) & \\
\hline \multirow[t]{2}{*}{ HDT } & & Mel & $288 / 605$ & $52(48-56)$ & $<0.001$ & $137 / 257$ & $48(41-54)$ & $151 / 348$ & $56(50-61)$ & 0.055 \\
\hline & & & $149 / 239$ & $39(33-45)$ & & $139 / 209$ & $35(29-42)$ & $10 / 30$ & $67(47-80)$ & \\
\hline \multicolumn{11}{|c|}{ Status Prior Maintenance } \\
\hline \multicolumn{3}{|c|}{ CR } & $225 / 468$ & $52(48-47)$ & 0.002 & $144 / 258$ & $46(39-52)$ & $81 / 210$ & $61(53-67)$ & 0.84 \\
\hline \multicolumn{3}{|c|}{ PR } & $70 / 109$ & $35(26-44)$ & & $42 / 57$ & $26(15-33)$ & $28 / 52$ & $45(32-58)$ & \\
\hline \multicolumn{3}{|c|}{ (B) Overall Survival } & Events/Pts & 5 -year OS (95\% CI) & $p$-value ${ }^{A}$ & Events/Pts & 5 -year OS (95\% CI) & Events/Pts & 5-year OS (95\% CI) & \\
\hline \multirow{2}{*}{\multicolumn{2}{|c|}{ Sex }} & female & $138 / 320$ & $59(53-64)$ & 0.536 & $95 / 180$ & $53(45-60)$ & $43 / 140$ & $59(53-64)$ & 0.488 \\
\hline & & male & $234 / 524$ & $55(50-59)$ & & $150 / 286$ & $49(43-55)$ & $84 / 238$ & $55(50-59)$ & \\
\hline \multirow{3}{*}{\multicolumn{2}{|c|}{ Age }} & $<1.5$ years & $45 / 119$ & $62(52-70)$ & 0.445 & $26 / 64$ & $61(48-72)$ & $19 / 55$ & $62(52-70)$ & 0.409 \\
\hline & & $1.5-<5$ years & $259 / 587$ & $57(53-61)$ & & $177 / 333$ & $50(45-55)$ & $82 / 254$ & $57(53-61)$ & \\
\hline & & $\geq 5$ years & $68 / 138$ & $49(40-58)$ & & $42 / 69$ & $42(30-54)$ & $26 / 69$ & $49(40-58)$ & \\
\hline \multirow{4}{*}{\multicolumn{2}{|c|}{ Stage }} & localised & $24 / 92$ & $76(66-83)$ & 0.001 & $13 / 60$ & $82(69-89)$ & $11 / 32$ & $76(66-83)$ & 0.003 \\
\hline & & Stage 4 & $347 / 745$ & $54(50-57)$ & & $232 / 406$ & $46(41-51)$ & $115 / 339$ & $54(50-57)$ & \\
\hline & & $\begin{array}{c}\text { Stage } 4 \\
\text { MNA no- }\end{array}$ & $193 / 414$ & $53(47-58)$ & 0.235 & $129 / 217$ & $43(37-50)$ & $64 / 197$ & $53(47-58)$ & 0.150 \\
\hline & & $\begin{array}{c}\text { Stage } 4 \\
\text { MNA yes+ }\end{array}$ & $137 / 288$ & $54(48-60)$ & & $88 / 162$ & $49(41-56)$ & $49 / 126$ & $54(48-60)$ & \\
\hline \multirow{5}{*}{\multicolumn{2}{|c|}{ MC }} & 0 & $24 / 92$ & $76(66-83)$ & $<0.001$ & $13 / 60$ & $82(69-89)$ & $11 / 32$ & $76(66-83)$ & 0.013 \\
\hline & & 1 & $36 / 105$ & $68(58-76)$ & & $29 / 70$ & $62(50-73)$ & $7 / 35$ & $68(58-76)$ & \\
\hline & & 2 & $109 / 248$ & $56(50-62)$ & & $71 / 136$ & $51(42-59)$ & $38 / 112$ & $56(50-62)$ & \\
\hline & & 3 & $117 / 232$ & $49(42-56)$ & & $81 / 120$ & $36(27-44)$ & $36 / 112$ & $49(42-56)$ & \\
\hline & & $>3$ & $70 / 121$ & $43(34-52)$ & & $41 / 57$ & $32(20-44)$ & $29 / 64$ & $43(34-52)$ & \\
\hline \multirow{2}{*}{\multicolumn{2}{|c|}{ TVD }} & no & $281 / 641$ & $57(53-61)$ & 0.224 & $200 / 391$ & $52(47-57)$ & $81 / 250$ & $57(53-61)$ & 0.441 \\
\hline & & yes & $78 / 170$ & $52(44-60)$ & & $\begin{array}{l}34 / 52 \\
34 / 5\end{array}$ & $36(23-49)$ & $44 / 118$ & $52(44-60)$ & \\
\hline \multirow{2}{*}{\multicolumn{2}{|c|}{ Surgery }} & CME & $248 / 578$ & $57(53-61)$ & 0.218 & $165 / 318$ & $51(45-56)$ & $83 / 260$ & $57(53-61)$ & 0.765 \\
\hline & & IME & $91 / 188$ & $52(45-59)$ & & $58 / 101$ & $46(36-55)$ & $33 / 87$ & $52(45-59)$ & \\
\hline \multicolumn{3}{|c|}{ Status Prior HDT } & & & & & & & & \\
\hline
\end{tabular}


Table 2. Cont

\begin{tabular}{|c|c|c|c|c|c|c|c|c|c|}
\hline & $C R$ & $116 / 290$ & $60(54-66)$ & 0.089 & $80 / 174$ & $56(48-63)$ & $36 / 116$ & $60(54-66)$ & 0.522 \\
\hline \multirow{4}{*}{ HDT } & VGPR & $140 / 308$ & $55(49-61)$ & & $86 / 159$ & $50(42-58)$ & $54 / 149$ & $55(49-61)$ & \\
\hline & PR & $92 / 188$ & $52(45-59)$ & & $65 / 108$ & $44(34-53)$ & $27 / 80$ & $52(45-59)$ & \\
\hline & BUMEL & $238 / 605$ & $60(56-64)$ & $<0.001$ & $121 / 257$ & $56(50-62)$ & $117 / 348$ & $60(56-64)$ & 0.267 \\
\hline & CEM & $134 / 239$ & $46(40-53)$ & & $124 / 209$ & $44(37-50)$ & $10 / 30$ & $46(40-53)$ & \\
\hline (B) $\mathrm{O}$ & & Events/Pts & 5 -year OS $(95 \% \mathrm{CI})$ & $p$-value ${ }^{\mathrm{A}}$ & Events/Pts & 5-year OS (95\% CI) & Events/Pts & 5-year OS $(95 \%$ CI) & \\
\hline \multicolumn{10}{|c|}{ Status Prior Maintenance } \\
\hline & $\mathrm{CR}$ & $190 / 468$ & $60(55-65)$ & 0.006 & $129 / 258$ & $54(47-59)$ & $61 / 210$ & $60(55-65)$ & 0.640 \\
\hline & VGPR & $83 / 192$ & $57(49-64)$ & & $47 / 93$ & $52(42-62)$ & $36 / 99$ & $57(49-64)$ & \\
\hline
\end{tabular}



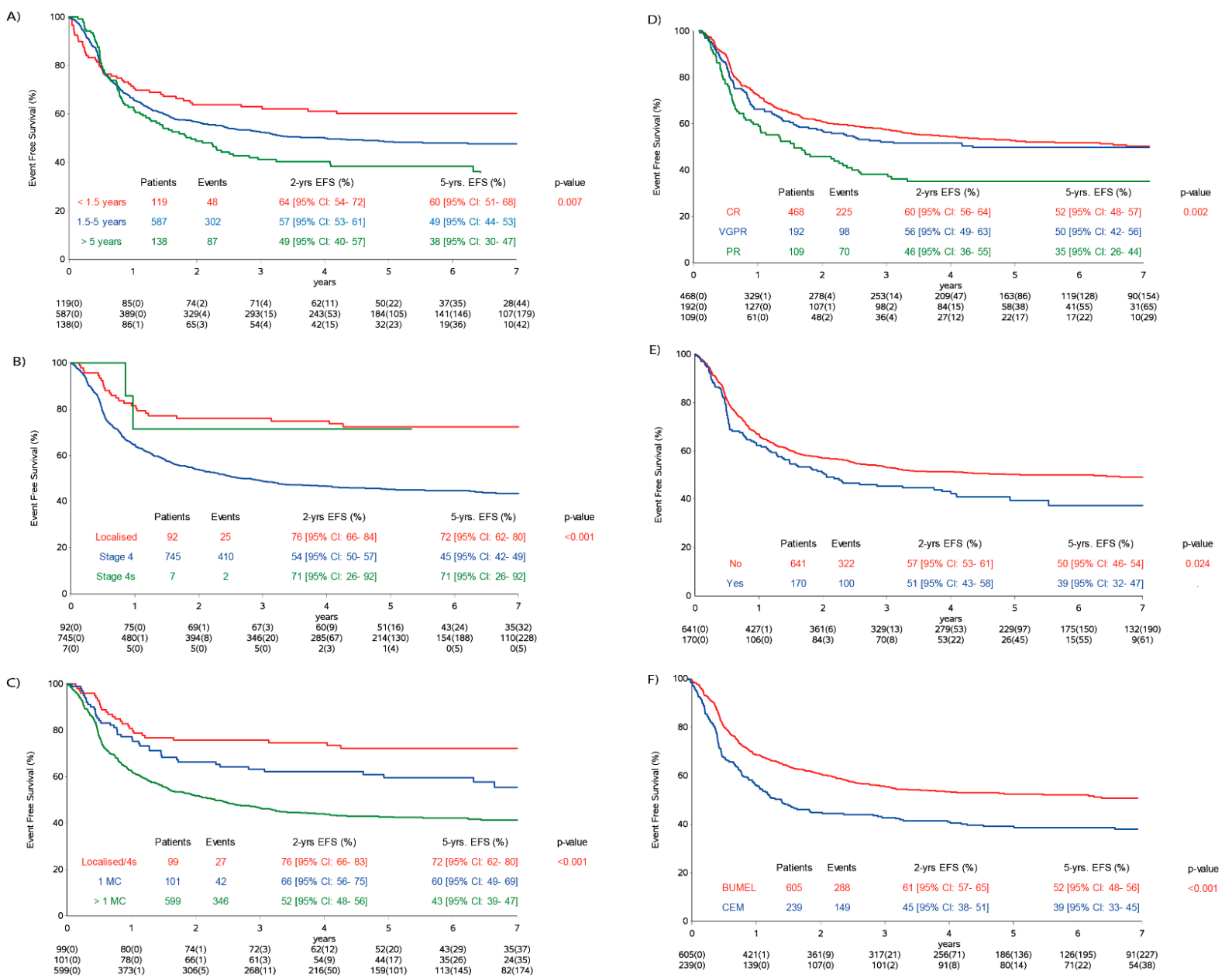

Figure 3. Influence of risk factors within the analysis population on event-free survival (EFS). (A) EFS and age; (B) EFS and stage; (C) EFS and metastatic compartments (MC) localised and 4s stage vs. stage 4 (one MC vs. multiple MC); (D) response status prior to maintenance phase: CR (complete remission), VGPR (very good partial remission) and PR (partial remission) and (E) EFS and TVD (topotecan, vincristine and doxorubicin). TVD added $=$ yes and TVD not added $=$ no. $(\mathbf{F})$ Type of high-dose chemotherapy $=$ BuMel (busulfan and melphalan) and CEM (carboplatin, etoposide and melphalan). 
In the IP, the two-year and five-year EFS rates for 210 patients (81 events) in complete remission (CR) were 68\% (95\% CI: 61-74\%) and 61\% (95\% CI: 53-57\%). In the CP, the two-year and five-year EFS rates for 258 patients in CR (144 events) were 54\% (95\% CI: 48-60\%) and 46\% (95\% CI: 39-52\%).

The impact of immunotherapy on EFS was significantly influenced by stage and MC, and the impact of immunotherapy was stronger in patients with metastatic disease (Table 2). Furthermore, a borderline significant interaction between maintenance treatment and HDT ( $p=0.055)$ was observed.

\subsection{Multivariate Analysis on Analysis Cohort}

Patients who had no immunotherapy ( $p=<.0001$, cumulative hazard ratio (cHR) 1.75) HDT with CEM ( $p=0.0345$, cHR 1.3); partial remission (PR) prior maintenance therapy ( $p=0.0103$, cHR 1.49); more than one MC at diagnosis $(p<0.001, \mathrm{cHR} 2.69)$ and age $>5$ years $(p=0.0138, \mathrm{cHR} 1.59)$ had a higher risk of relapse (Table 3).

Table 3. Multivariate analysis of the analysis cohort. cHR (cumulative hazard ratios); $95 \% \mathrm{CI}$ ( $95 \%$ confidence interval); $p$-value (probability value); MNA (MYCN amplification); MC (metastatic compartments, referring either to bone marrow, skeletal or lymph node involvement); TVD (topotecan, vincristine and doxorubicin); CR (complete remission); VGPR (very good partial remission); PR (partial remission); HDT (high-dose chemotherapy); BuMel (high-dose chemotherapy with busulfan and melphalan); CEM (high-dose chemotherapy with carboplatin, etoposide and melphalan); IP (immunotherapy population) and CP (control population). * test for the global main effect for risk-factors with more than two categories.

\begin{tabular}{|c|c|c|c|c|}
\hline & \multirow{2}{*}{ Risk Factor } & \multirow{2}{*}{ Characteristics } & \multicolumn{2}{|c|}{ Pseudo Values for 5-Years EFS } \\
\hline & & & cHR $(95 \%$ CI) & $p$-Value \\
\hline \multirow[t]{13}{*}{ (A) } & \multicolumn{4}{|c|}{ Multivariate Analysis } \\
\hline & \multicolumn{2}{|c|}{ Immunotherapy vs. Control Cohort } & $1.75(1.36-2.25)$ & $<0.0001$ \\
\hline & \multirow[t]{3}{*}{ Age (vs. $<1.5 \mathrm{yr}$} & & & 0.0931 * \\
\hline & & $1.5-5$ years & $1.31(0.92-1.87)$ & 0.1384 \\
\hline & & $>5$ years & $1.59(1.05-2.42)$ & 0.0138 \\
\hline & \multicolumn{3}{|c|}{$\begin{array}{l}\text { Stage } 4,4 \text { s and Number of MC (vs. } \\
\text { MNA stages } 2,3 \text { ) }\end{array}$} & $<0.0001 *$ \\
\hline & & $1 \mathrm{MC}$ & $1.38(0.80-2.47)$ & 0.2493 \\
\hline & & $>1 \mathrm{MC}$ & $2.69(1.74-4.15)$ & $<0.0001$ \\
\hline & TVD & & $1.28(0.97-1.69)$ & 0.2478 \\
\hline & \multicolumn{3}{|c|}{ Status Prior Maintenance (vs. CR) } & $0.0363 *$ \\
\hline & & VGPR & $1.06(0.81-1.39)$ & 0.6416 \\
\hline & & PR & $1.49(1.10-2.02)$ & 0.0103 \\
\hline & HDT & CEM vs. BuMel & $1.32(1.02-1.70)$ & 0.0345 \\
\hline \multirow[t]{3}{*}{ (B) } & \multicolumn{4}{|c|}{$\begin{array}{l}\text { Subgroup Analysis According to HDT(after adjustment for age, stage, MC, TVD } \\
\text { and status prior maintenance treatment) }\end{array}$} \\
\hline & BUMEL & IP vs. CP & $1.6(1.2-2.1)$ & 0.001 \\
\hline & CEM & IP vs. $\mathrm{CP}$ & $3.0(1.5-5.8)$ & 0.002 \\
\hline
\end{tabular}


After adjustment for age, stage, MC, TVD and response prior to maintenance therapy (Table 3), a benefit from immunotherapy was confirmed for either HDT (busulfan and melphalan (BuMel) or CEM). Patients receiving BuMel had an adjusted cumulative hazard ratio of $1.6(1.2-2.1)$ with an unadjusted five-year EFS for the IP of 56\% (95\% CI: 50-61\%) and 48\% (95\% CI: 41-54\%) for the CP $(p=0.001)$. Patients receiving CEM had an adjusted cumulative hazard ratio of $3.0(1.5-5.8)$ and showed an unadjusted five-year EFS of the IP of $67 \%$ (95\% CI: $47-80 \%$ ) versus 35\% (95\% CI: $29-42 \%)$ for the CP $(p=0.002)$.

\subsection{Response to Maintenance Treatments}

Thirty-nine of one hundred and eight (36\%) CP patients with evaluable diseases prior to maintenance (67 very good partial remission (VGPR) and 41 partial remission (PR)) responded; of whom, 35/108 (32\%) achieved CR after isotretinoin. In contrast, 64/130 (49\%) of IP patients with evaluable diseases (85 VGPR and 45 PR) prior to immunotherapy responded; of whom, 52/130 (40\%) achieved CR after immunotherapy (Table $4, p=0.226$ ).

Table 4. Response for immunotherapy and control populations. Legend: CR (complete remission), VGPR (very good partial remission), PR (partial remission), SD (stable disease) and PD (progressing disease).

\begin{tabular}{|c|c|c|c|c|c|c|c|c|}
\hline \multicolumn{3}{|c|}{ Response Status before Maintenance } & \multicolumn{6}{|c|}{ Response Status after Maintenance } \\
\hline & & \multirow[t]{2}{*}{ Total } & Evaluable & CR & VGPR & PR & SD & PD \\
\hline & & & & \multicolumn{5}{|c|}{ Immunotherapy and Isotretinoin } \\
\hline CR & & 210 & 188 & 151 & 0 & 0 & $\mathbf{0}$ & 37 \\
\hline \multirow[t]{4}{*}{$<\mathrm{CR}$} & Total & 151 & 130 & 52 & 43 & 8 & 0 & 27 \\
\hline & VGPR & 99 & 85 & 36 & 31 & 0 & 0 & 18 \\
\hline & PR & 52 & 45 & 16 & 12 & 8 & 0 & 9 \\
\hline & & & & \multicolumn{5}{|c|}{ Isotretinoin } \\
\hline CR & & 258 & 204 & 163 & 0 & 0 & 0 & 40 \\
\hline \multirow[t]{3}{*}{$<\mathrm{CR}$} & Total & 150 & 108 & 35 & 27 & 18 & 1 & 27 \\
\hline & VGPR & 93 & 67 & 28 & 23 & 2 & 0 & 14 \\
\hline & PR & 57 & 41 & 7 & 4 & 16 & 1 & 13 \\
\hline
\end{tabular}

\subsection{Adverse Events and Toxicity}

Adverse events (CTC Grades 1 to 4) are summarized in Table 5. Toxicity tended to be higher in the IP population, particularly in those patients who received sc-IL-2. Four patients had non-relapse-related mortality in the IP and four in the CP. 
Table 5. Toxicities IL-2 (interleukin 2), non-hem. tox. (non-hematological toxicities), WBC (white blood cells), ECHO LV (left ventricular)/SV (stroke volume), GFR (glomerular filtration rate), central neuro (central neurotoxicity), periph neuro (peripheral neurotoxicity) and liver enzymes: SGOT (serum-glutamat-oxalacetat-transaminase)/SGPT (serum-glutamat-pyruvat-transaminase). Columns in bold show the values for the combined grade 3 and 4 toxicities.

\begin{tabular}{|c|c|c|c|c|c|c|c|c|c|c|c|c|c|c|c|c|c|c|c|c|c|c|c|c|}
\hline \multirow[t]{2}{*}{ Toxicities } & \multicolumn{8}{|c|}{ Control } & \multicolumn{8}{|c|}{ Without IL2 } & \multicolumn{8}{|c|}{ With IL2 } \\
\hline & \multirow{2}{*}{$\begin{array}{c}\text { Eval } \\
317\end{array}$} & \multirow{2}{*}{\begin{tabular}{|c|}
$\mathbf{0}$ \\
113
\end{tabular}} & \multirow{2}{*}{$\begin{array}{c}\mathbf{1} \\
42\end{array}$} & \multirow{2}{*}{$\begin{array}{c}2 \\
116\end{array}$} & \multirow{2}{*}{$\begin{array}{c}3 \\
41\end{array}$} & \multirow{2}{*}{$\begin{array}{l}4 \\
5\end{array}$} & \multicolumn{2}{|c|}{$3+4$} & \multirow{2}{*}{$\begin{array}{l}\text { All } \\
186\end{array}$} & \multirow{2}{*}{$\begin{array}{l}\mathbf{0} \\
5\end{array}$} & \multirow{2}{*}{$\begin{array}{l}1 \\
6\end{array}$} & \multirow{2}{*}{$\begin{array}{c}2 \\
53\end{array}$} & \multirow{2}{*}{$\begin{array}{c}3 \\
105\end{array}$} & \multirow{2}{*}{$\begin{array}{c}4 \\
17\end{array}$} & \multicolumn{2}{|c|}{$3+4$} & \multirow{2}{*}{$\begin{array}{l}\text { All } \\
192\end{array}$} & \multirow{2}{*}{$\begin{array}{l}\mathbf{0} \\
4\end{array}$} & 1 & 2 & 3 & 4 & & \\
\hline Non-Hem. Tox. & & & & & & & 46 & $15 \%$ & & & & & & & 122 & $66 \%$ & & & 2 & 20 & 113 & 53 & 166 & $86 \%$ \\
\hline General Condition & 314 & 225 & 69 & 13 & 4 & 3 & 7 & $2 \%$ & 185 & 42 & 70 & 43 & 24 & 6 & 30 & $16 \%$ & 192 & 25 & 53 & 36 & 66 & 12 & 78 & $41 \%$ \\
\hline Haemoglobin & 313 & 208 & 39 & 54 & 8 & 4 & 12 & $4 \%$ & 186 & 21 & 2 & 84 & 69 & 10 & 79 & $42 \%$ & 191 & 14 & 5 & 46 & 102 & 24 & 126 & $66 \%$ \\
\hline WBC & 313 & 235 & 32 & 28 & 15 & 3 & 18 & $6 \%$ & 186 & 35 & 30 & 73 & 42 & 6 & 48 & $26 \%$ & 191 & 35 & 30 & 57 & 51 & 18 & 69 & $36 \%$ \\
\hline Granulocytes & 313 & 244 & 23 & 25 & 16 & 5 & 21 & $7 \%$ & 186 & 44 & 26 & 54 & 43 & 19 & 62 & $33 \%$ & 191 & 34 & 12 & 34 & 69 & 42 & 111 & $58 \%$ \\
\hline Platelets & 313 & 260 & 13 & 12 & 16 & 12 & 28 & $9 \%$ & 186 & 66 & 25 & 31 & 40 & 24 & 64 & $34 \%$ & 191 & 30 & 18 & 26 & 61 & 56 & 117 & $61 \%$ \\
\hline Infection & 315 & 220 & 52 & 23 & 19 & 1 & 20 & $6 \%$ & 185 & 79 & 26 & 32 & 47 & 1 & 48 & $26 \%$ & 191 & 60 & 20 & 47 & 58 & 6 & 64 & $34 \%$ \\
\hline Fever & 314 & 241 & 14 & 54 & 4 & 1 & 5 & $2 \%$ & 185 & 41 & 3 & 116 & 24 & 1 & 25 & $14 \%$ & 190 & 28 & 4 & 82 & 66 & 10 & 76 & $40 \%$ \\
\hline Stomatitis & 312 & 293 & 10 & 7 & 2 & 0 & 2 & $1 \%$ & 185 & 156 & 18 & 8 & 0 & 3 & 3 & $2 \%$ & 191 & 149 & 27 & 12 & 2 & 1 & 3 & $2 \%$ \\
\hline Nausea/Vomiting & 313 & 284 & 6 & 19 & 4 & 0 & 4 & $1 \%$ & 185 & 88 & 11 & 76 & 9 & 1 & 10 & $5 \%$ & 191 & 68 & 12 & 94 & 14 & 3 & 17 & $9 \%$ \\
\hline Diarrhoea & 313 & 286 & 11 & 13 & 3 & 0 & 3 & $1 \%$ & 185 & 93 & 32 & 47 & 10 & 3 & 13 & $7 \%$ & 192 & 75 & 25 & 51 & 34 & 7 & 41 & $21 \%$ \\
\hline Constipation & 312 & 302 & 7 & 3 & 0 & 0 & 0 & $0 \%$ & 185 & 110 & 43 & 32 & 0 & 0 & 0 & $0 \%$ & 191 & 142 & 21 & 21 & 4 & 3 & 7 & $4 \%$ \\
\hline Skin & 315 & 181 & 50 & 73 & 10 & 1 & 11 & $3 \%$ & 185 & 65 & 46 & 65 & 9 & 0 & 9 & $5 \%$ & 192 & 48 & 48 & 77 & 19 & 0 & 19 & $10 \%$ \\
\hline Allergy & 314 & 307 & 4 & 3 & 0 & 0 & 0 & $0 \%$ & 185 & 88 & 50 & 28 & 14 & 5 & 19 & $10 \%$ & 191 & 75 & 39 & 38 & 32 & 7 & 39 & $20 \%$ \\
\hline Cardiac Function & 298 & 298 & 0 & 0 & 0 & 0 & 0 & $0 \%$ & 182 & 178 & 0 & 0 & 3 & 1 & 4 & $2 \%$ & 191 & 183 & 3 & 1 & 3 & 1 & 4 & $2 \%$ \\
\hline Echo LV/SV & 298 & 298 & 0 & 0 & 0 & 0 & 0 & $0 \%$ & 182 & 181 & 0 & 0 & 0 & 1 & 1 & $1 \%$ & 189 & 181 & 5 & 2 & 0 & 1 & 1 & $1 \%$ \\
\hline Hypotension & 298 & 296 & 2 & 0 & 0 & , & 0 & $0 \%$ & 182 & 139 & 22 & 8 & 12 & 1 & 13 & $7 \%$ & 191 & 119 & 23 & 17 & 25 & 7 & 32 & $17 \%$ \\
\hline Hypertension & 298 & 298 & 0 & 0 & 0 & 0 & 0 & $0 \%$ & 182 & 162 & 10 & 3 & 7 & 0 & 7 & $4 \%$ & 190 & 177 & 4 & 6 & 3 & 0 & 3 & $2 \%$ \\
\hline Creatinine & 312 & 301 & 9 & 2 & 0 & 0 & 0 & $0 \%$ & 185 & 167 & 14 & 1 & 3 & 0 & 3 & $2 \%$ & 192 & 159 & 20 & 11 & 2 & 0 & 2 & $1 \%$ \\
\hline Proteinuria & 311 & 307 & 4 & 0 & 0 & 0 & 0 & $0 \%$ & 184 & 169 & 13 & 2 & 0 & 0 & 0 & $0 \%$ & 191 & 178 & 12 & 1 & 0 & 0 & 0 & $0 \%$ \\
\hline Haematuria & 311 & 305 & 6 & 0 & 0 & 0 & 0 & $0 \%$ & 183 & 167 & 11 & 5 & 0 & 0 & 0 & $0 \%$ & 191 & 169 & 16 & 6 & 0 & 0 & 0 & $0 \%$ \\
\hline GFR & 310 & 302 & 5 & 3 & 0 & 0 & 0 & $0 \%$ & 183 & 172 & 6 & 2 & 3 & 0 & 3 & $2 \%$ & 190 & 179 & 9 & 1 & 1 & 0 & 1 & $1 \%$ \\
\hline Central Neu & 311 & 304 & 4 & 0 & 0 & 3 & 3 & $1 \%$ & 185 & 165 & 14 & 3 & 3 & 0 & 3 & $2 \%$ & 191 & 158 & 16 & 6 & 3 & 8 & 11 & $6 \%$ \\
\hline Periph Neuro & 311 & 308 & 1 & 0 & 1 & 1 & 2 & $1 \%$ & 185 & 173 & 8 & 3 & 1 & 0 & 1 & $1 \%$ & 191 & 167 & 14 & 4 & 5 & 1 & 6 & $3 \%$ \\
\hline Bilirubin & 309 & 301 & 4 & 2 & 2 & 0 & 2 & $1 \%$ & 185 & 169 & 1 & 10 & 4 & 1 & 5 & $3 \%$ & 192 & 159 & 5 & 21 & 6 & 1 & 7 & $4 \%$ \\
\hline SGOT/SGPT & 311 & 218 & 68 & 19 & 6 & 0 & 6 & $2 \%$ & 185 & 68 & 43 & 43 & 30 & 1 & 31 & $17 \%$ & 192 & 68 & 40 & 40 & 43 & 1 & 44 & $23 \%$ \\
\hline Dilated Pupils & 22 & 22 & 0 & 0 & 0 & 0 & 0 & $0 \%$ & 123 & 108 & 15 & 0 & 0 & 0 & 0 & $0 \%$ & 125 & 95 & 30 & 0 & 0 & 0 & 0 & $0 \%$ \\
\hline Accommodation Defects & 22 & 22 & 0 & 0 & 0 & 0 & 0 & $0 \%$ & 121 & 115 & 6 & 0 & 0 & 0 & 0 & $0 \%$ & 125 & 111 & 14 & 0 & 0 & 0 & 0 & $0 \%$ \\
\hline Capillary Leak Syndrome & 19 & 18 & 0 & 1 & 0 & 0 & 0 & $0 \%$ & 119 & 91 & 0 & 23 & 5 & 0 & 5 & $4 \%$ & 124 & 70 & 0 & 35 & 16 & 3 & 19 & $15 \%$ \\
\hline Cytokine Release Syndrome & 19 & 18 & 1 & 0 & 0 & 0 & 0 & $0 \%$ & 118 & 95 & 8 & 10 & 5 & 0 & 5 & $4 \%$ & 123 & 85 & 12 & 17 & 9 & 0 & 9 & $7 \%$ \\
\hline Pain related to ch14.18/CHO & & & & & & & & & 122 & 42 & 17 & 44 & 19 & 0 & 19 & $16 \%$ & 124 & 28 & 22 & 42 & 31 & 1 & 32 & $26 \%$ \\
\hline Papilloedema & 22 & 22 & 0 & 0 & 0 & 0 & 0 & $0 \%$ & 120 & 113 & 7 & 0 & 0 & 0 & 0 & $0 \%$ & 123 & 121 & 2 & 0 & 0 & 0 & 0 & $0 \%$ \\
\hline
\end{tabular}




\section{Discussion}

This analysis showed superior EFS and OS in the era when dinutuximab beta-based immunotherapy was included in therapy for high-risk neuroblastoma, compared to the previous era in the same trial when isotretinoin alone was the only element of maintenance therapy. Although there are limitations with a historical comparison, this is the first and possibly only demonstration that the addition of dinutuximab beta as immunotherapy improves survival in the high-risk neuroblastoma front-line population treated homogenously in the HR-NBL1/SIOPEN trial. Both the control and immunotherapy populations received the same treatment approach. Furthermore, this analysis is an important contribution, as data on the efficacy of anti-GD $\mathrm{GD}_{2}$ antibody-based immunotherapy are limited to one prospective randomised trial and a few retrospective analyses. Ethical concerns precluded a randomised comparison of immunotherapy after the results of the COG ANBL0032 trial emerged. A randomised trial of dinutuximab beta and isotretinoin compared to isotretinoin alone would have produced more robust data, but this was believed not to be ethically feasible within the SIOPEN community.

As the two cohorts were from the same trial and using the same criteria; in particular, HDT within nine months from diagnosis and no progression at 109 days after ASCR as a starting point for survival, with an unchanged supportive care protocol guidance, this analysis provides important data supporting the benefit of dinutuximab beta-based immunotherapy. The landmark time identified was the median time observed between ASCR and initiation of dinutuximab beta; thus, only patients without progressive diseases at this timepoint were included in the pre-immunotherapy $\mathrm{CP}$. The introduction of this landmark was important in order to exclude early relapse before immunotherapy could be commenced. Both populations, IP and CP, were balanced for age, sex, stage 4, MYCN amplification and response prior to HDT.

The benefit of immunotherapy was further underpinned by multivariate analysis. After adjustment for risk factors (for example, age, stage, MC at diagnosis, need for TVD and response prior to maintenance therapy) a positive impact on outcome was observed with either BuMel or CEM. This is particularly important in view of a higher percentage of patients receiving CEM in the control group, as we previously have shown superior outcomes for patients treated with BuMel [6]. Hence, the superior outcomes in the immunotherapy group are unlikely to be solely related to BuMel. These conclusions are supported by the recent publication from COG [13] demonstrating that immunotherapy improves survival even after optimised HDT regimens.

Response prior to immunotherapy is an important prognostic factor. Patients treated in CR in the IP had a two-year EFS of $68 \%$, compared to $54 \%$ in the CP. Acknowledging that comparisons across trials are challenging, these results are similar to the previous report of dinutuximab in combination with IL-2 and GM-CSF in patients in CR, resulting in a two-year EFS of $66 \%$ for patients treated by immunotherapy, compared to only $46 \%$ in patients with an isotretinoin maintenance treatment [7]. Further support in favour of dinutuximab beta-based immunotherapy within the HR-NB11/SIOPEN trial comes from improved response rates in patients with residual diseases at the site of the primary tumour or metaiodobenzylguanidine (mIBG)-positive skeletal disease following immunotherapy; a $49 \%$ response rate and a $40 \%$ CR rate was observed in patients treated with immunotherapy, as compared to a $36 \%$ overall response and $32 \% \mathrm{CR}$ rate in the control population.

The increasing role for immunotherapy, with anti-GD $\mathrm{GD}_{2}$ antibodies, in the therapy of patients with high-risk neuroblastoma is further highlighted by the recent demonstration of the efficacy of combining anti-GD 2 antibodies with chemotherapy, either at relapse or at initial presentation $[14,15]$.

In summary, this report describes the effects of including dinutuximab beta in high-risk neuroblastoma maintenance therapy and shows a clear survival benefit. This provides an important baseline to further build immunotherapy strategies in this challenging patient population. 


\section{Materials and Methods}

\subsection{Trial Eligibility}

HR-NBL1/SIOPEN, an international, randomised, multiarm, open-label, phase 3 trial for high-risk neuroblastoma, opened on 24 June 2002 and is registered with ClinTrials.gov, number NCT01704716, and EudraCT, number 2006-001489-17. All randomisations of the HR-NBL1/SIOPEN trial are closed, and three have been published $[5,6,12]$. SIOPEN institutions recruited patients after approval of the trial by national regulatory authorities and ethical committees. Parents/guardians and patients provided written informed consent or assent, when applicable.

The International Neuroblastoma Staging System criteria (INSS) and International Neuroblastoma Response Criteria (INRC) [16] were used to classify the disease and to evaluate responses to therapy. Untreated patients with INSS stage 4 metastatic neuroblastoma aged 1-20 years or INSS stage 2-4 neuroblastoma with MYCN amplification, as determined in SIOPEN reference laboratories [17], any age up to 20 years, were eligible. The SIOPEN-R-NET web-based system (https://www.siopen-r-net.org/) randomly assigned eligible patients in real-time.

\subsection{Eligibiliy for the Analysis Cohort and Treatments Given}

This analysis included all patients registered in the HR-NBL1/SIOPEN trial between 2002 and 2013 who met the criteria: (i) HDT within 9 months from diagnosis and (ii) no progression at 109 days after ASCR. The median time between ASCR and initiation of dinutuximab beta was 109 days; therefore, only patients without progressive diseases at this landmark timepoint were included in the pre-immunotherapy control population $(\mathrm{CP})$. The 18 patients randomised to receive dinutuximab beta and isotretinoin between 2006 and 2009 were excluded from this analysis. Two cohorts were compared, a CP between 2002-2009 who did not receive dinutuximab beta and an IP between 2009 and 2013 who was randomised to receive dinutuximab beta with or without sc-IL2 [12]. As the addition of IL-2 to immunotherapy with dinutuximab beta did not improve outcome, both randomised arms were considered as one group for the purposes of this analysis [12]. The CP comprised patients who received HDT randomised to BuMel or CEM [6] but did not receive immunotherapy.

All patients received rapid COJEC induction [4,5] (Figure 4). Between 2002 to 2010, patients who had a bone marrow complete response (CR) and a metastatic CR or a metastatic partial response (PR) defined by INSS criteria but at least 50\% reduction in skeletal metaiodobenzylguanidine (mIBG) positivity from baseline and three or fewer areas of abnormal uptake on ${ }^{123} \mathrm{I}-\mathrm{mIBG}$ scintigraphy were eligible for HDT randomisation comparing BuMel with CEM [6]. Thereafter, BuMel became the standard of care. Patients who did not achieve a metastatic response to fulfil the HDT eligibility criteria received two courses of topotecan, vincristine and doxorubicin (TVD) [18] prior to HDT. Local treatment of the primary tumour comprised attempted total surgical resection and radiotherapy (21 Gy) to the primary tumour site between 60 and 90 days after ASCR. There was no dose modification in the event of incomplete tumour excision; neither were metastatic sites systematically irradiated. 


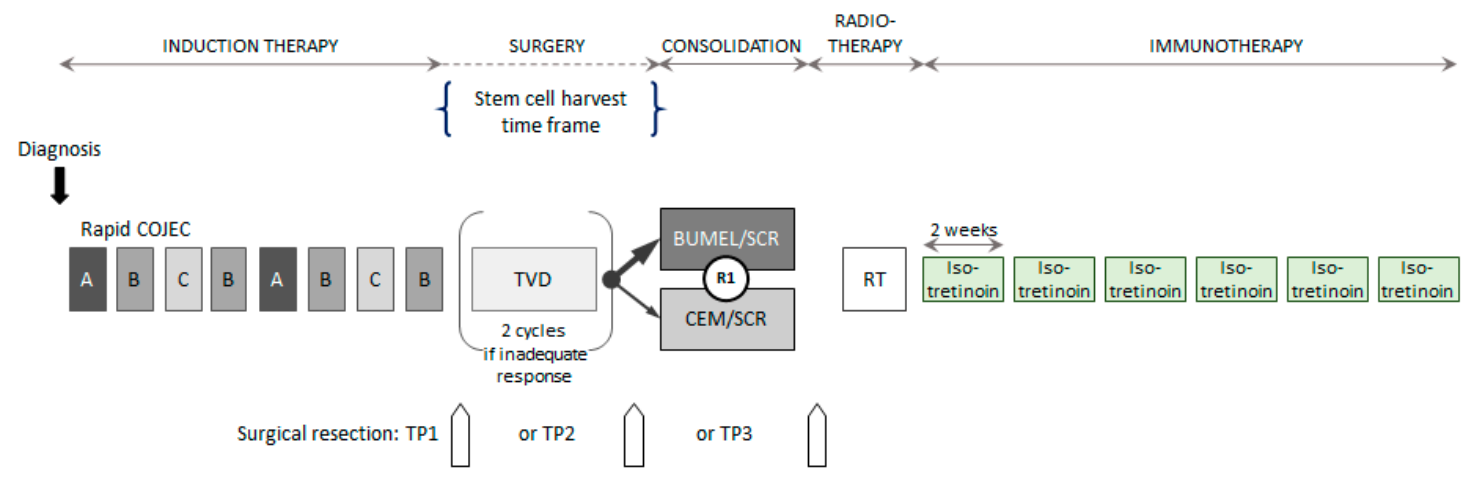

I

Rapid COJEC

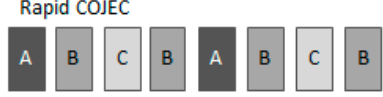
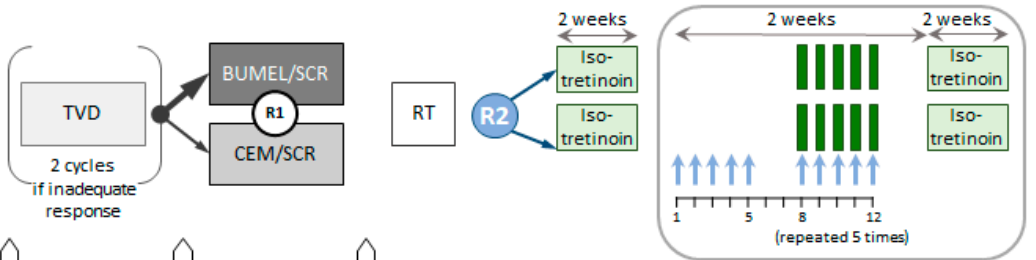

Surgical resection: TP1 $\ \quad$ or TP2 $\bigsqcup \quad$ or TP3

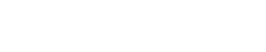

(repeated 5 times)

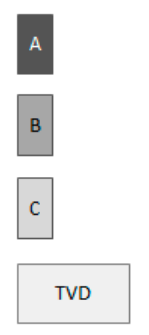

\begin{tabular}{|c|c|c|}
\hline Course A & $\begin{array}{l}\text { Vincristine } \\
\text { Carboplatin } \\
\text { Etoposide }\end{array}$ & $\begin{array}{l}1.5 \mathrm{mg} / \mathrm{m}^{2} \text { (maximum dose } 2 \mathrm{mg} \text { ) } \times 1 \text { day } \\
750 \mathrm{mg} / \mathrm{m}^{2} \times 1 \text { day } \\
175 \mathrm{mg} / \mathrm{m}^{2} \times 2 \text { days }\end{array}$ \\
\hline Course B & $\begin{array}{l}\text { Vincristine } \\
\text { Cisplatin }\end{array}$ & $\begin{array}{l}1.5 \mathrm{mg} / \mathrm{m}^{2}(\text { maximum dose } 2 \mathrm{mg}) \times 1 \text { day } \\
80 \mathrm{mg} / \mathrm{m}^{2} / \mathrm{ctn} \text {. over } 24 \text { hours }\end{array}$ \\
\hline Course C & $\begin{array}{l}\text { Vincristine } \\
\text { Etoposide } \\
\text { Cyclophosphamide }\end{array}$ & $\begin{array}{l}1.5 \mathrm{mg} / \mathrm{m}^{2} \text { (maximum dose } 2 \mathrm{mg} \text { ) } \times 1 \text { day } \\
175 \mathrm{mg} / \mathrm{m}^{2} \times 2 \text { days } \\
1050 \mathrm{mg} / \mathrm{m}^{2} \times 2 \text { days }\end{array}$ \\
\hline $\begin{array}{l}\text { Altered } \\
\text { chemotherapy } \\
\text { (optional } 2 \text { cycles) }\end{array}$ & $\begin{array}{l}\text { Topotecan } \\
\text { Vincristine } \\
\text { Doxorubicin }\end{array}$ & $\begin{array}{l}1,5 \mathrm{mg} / \mathrm{m}^{2} \times 5 \text { days } \\
2 \mathrm{mg} / \mathrm{m}^{2} / \mathrm{ctn} \text {. over } 48 \text { hours } \\
50 \mathrm{mg} / \mathrm{m}^{2} / \mathrm{ctn} \text {. over } 48 \text { hours }\end{array}$ \\
\hline
\end{tabular}

\begin{tabular}{|c|c|c|c|}
\hline BUMEL/SCR ${ }^{11}$ & BUMEL & $\begin{array}{l}\text { Busilvex } \\
\text { Melphalan }\end{array}$ & $\begin{array}{l}<9 \mathrm{~kg}: 1 \mathrm{mg} / \mathrm{kg} ; 9 \mathrm{~kg} \text { to }<16 \mathrm{~kg}: 1.2 \mathrm{mg} / \mathrm{kg} ; 16 \mathrm{~kg} \text { to } 23 \mathrm{~kg} 1.1 \mathrm{mg} / \mathrm{kg} ;>23 \mathrm{~kg} \text { to } 34 \mathrm{~kg}: 0.95 \mathrm{mg} / \mathrm{kg} ;>34 \mathrm{~kg}: 0.8 \mathrm{mg} / \mathrm{kg} \mathrm{x} 5 \text { days } \\
140 \mathrm{mg} / \mathrm{m}^{2} \times 1 \text { day }\end{array}$ \\
\hline \multirow[t]{2}{*}{ CEM/SCR ${ }^{1]}$} & CEM & $\begin{array}{l}\text { Carboplatin } \\
\text { Etoposide } \\
\text { Melphalan }\end{array}$ & $\begin{array}{l}\text { AUC } 4.1 \mathrm{mg} / \mathrm{ml} . \mathrm{min} / \text { day } x 4 \text { days (based on the GFR rate) } \\
\leq 12 \mathrm{~kg}: 11.3 \mathrm{mg} / \mathrm{kg} / \text { day; }>12 \mathrm{~kg}: 338 \mathrm{mg} / \mathrm{m}^{2} / \text { day } \times 4 \text { days } \\
\leq 12 \mathrm{~kg}: 2.3 \mathrm{mg} / \mathrm{kg} / \text { day; }>12 \mathrm{~kg}: 70 \mathrm{mg} / \mathrm{m}^{2} / \text { day } 3 \text { days }\end{array}$ \\
\hline & \multicolumn{3}{|c|}{ 1) Stem Cell Reinfusion } \\
\hline RT & Radiotherapy & & Fractionated radiotherapy $(21 \mathrm{GY})$ given in 14 fractions of $1.5 \mathrm{~Gy}$ over not more than 21 days \\
\hline \begin{tabular}{|c|}
$150-$ \\
tretinoin
\end{tabular} & Retinoic Acid & Isotretinoin & $\begin{array}{l}160 \mathrm{mg} / \mathrm{m} / \text { day divided into two equal doses } \\
\text { given orally twice a day for } 14 \text { days }\end{array}$ \\
\hline & Immunotherapy & Ch14.18/CHO & $\begin{array}{l}20 \mathrm{mg} / \mathrm{m}^{2} / \mathrm{day}{ }^{2} \text { in } 8 \text { hours } \times 5 \text { days } \\
\text { 2) Infants and children with a body weight below } 12 \mathrm{~kg} \text { willbe dosed at } 0.67 \mathrm{mg} / \mathrm{kg} / \mathrm{day} \text {. In infants } \\
\text { weighing } \leq \text { to } 5 \mathrm{~kg} \text {, a further } 1 / 3 \text { dosereduction is advised }\end{array}$ \\
\hline$\uparrow \uparrow \uparrow \uparrow \uparrow$ & & Aldesleukin (II-2) & $6 \mathrm{MIU} / \mathrm{m} 2 /$ day subcutaneously on 5 consecutive days over 2 weeks \\
\hline
\end{tabular}

00

Possible time points for surgical resection

(1)

R1 - High dose therapy randomisation

R2-ch14.18/CHO based immunotherapy randomisation

Figure 4. HR-NBL1/SIOPEN treatment overview.

All patients received 6 cycles of oral isotretinoin over two weeks [3] after local irradiation. From 2009, patients were randomised between day 60 to 90 after ASCR to receive five courses of dinutuximab beta at a dose of $20 \mathrm{mg} / \mathrm{m}^{2} /$ day as an $8 \mathrm{~h}$ infusion for 5 consecutive days (total dose of $100 \mathrm{mg} / \mathrm{m}^{2}$ per cycle, days 8 to 12) with or without $6 \times 10^{6} \mathrm{IU} / \mathrm{m}^{2} /$ day of sc-IL-2 on days 1 to 5 and days 8 to 12 of each immunotherapy cycle [12]. 
Patients had full disease evaluations prior to and after 2 and 5 courses of maintenance treatment. This included whole body ${ }^{123} \mathrm{I}-\mathrm{mIBG}$ scintigraphy, CT or MRI scans of the primary tumour and any other evaluable site of the disease; bone marrow examination with both aspirates and trephines obtained from two sites and measurement of urinary catecholamine metabolites. Response was assessed by the 1993 INRC based on local institution reporting. The only evaluable diseases prior to immunotherapy were mIBG-positive skeletal disease or diseases detectable on CT/MRI scans prior to randomisation, as patients with bone marrow involvement were not eligible.

\subsection{Statistical Analysis}

\subsubsection{Establishment of the Analysis Cohort}

The number of MC at diagnosis was calculated according to the number of $\mathrm{MC}$ at diagnosis either in the bone marrow, skeleton or other sites, with a possible range from one to six. Characteristics of the $\mathrm{CP}$ were compared to the IP by the chi-square test to assess the balance of risk factors between the two populations.

\subsubsection{Outcome Parameters}

Follow-up commenced at 109 days for the CP cohort and from the first dose of dinutuximab beta for the IP cohort. EFS and OS were estimated using the Kaplan and Meier method and compared with the log-rank test [19]. CIR was estimated [20], taking into account the competing risk of death without relapse/progression. For cumulative incidence of non-relapse mortality, relapse or progression was considered as a competing event. The statistical comparison of cumulative incidences used Gray's methodology [21]. EFS, OS, cumulative incidence of relapse/progression and cumulative incidence of non-relapse mortality are presented as 5-year point estimates with confidence intervals (CI), as previously described [20,22]. Two-year EFS was determined to facilitate comparisons with published data [7]. $p$-values of less than 0.05 were considered to indicate statistical significance. In spite of the limitations of subgroup analysis (including multiple testing and lack of power), the cohorts were assessed according to baseline, pre and post-HDT risk factors (Table 2). A formal test for interaction was performed within a Cox model.

\subsubsection{Multivariate Analysis}

Multivariate analysis of treatment and risk factors was undertaken on the analysis cohort. In the presence of nonproportional hazards, as detected for age and MYCN amplification, the pseudo-value regression [22] for a 5-year EFS approach was chosen. The aim of this analysis was to adjust the comparison between the two populations (IP and CP) for potential confounders and risk factors, such as age, stage, addition of TVD, disease status prior to maintenance and HDT/ASCR. Using the same approach, a subgroup analysis was performed in order to separately evaluate the value of immunotherapy in patients with BuMel and CEM.

The data cut-off time of this analysis was July 31, 2017. Median follow-up was calculated using the inverse Kaplan Meier estimate. The statistical evaluation and power calculation were done with SAS 9.4 and Module LR1 of Pass 2002, respectively. 


\section{Conclusions}

This report shows that the introduction of dinutuximab beta is associated with a survival benefit for children with high-risk neuroblastoma. Similar results were reported for dinutuximab in one randomized trial and one nonrandomized investigation [23-25]. However, dinutuximab beta is a different molecule with a separate development pathway, and the demonstration of its beneficial effects on treatment outcome is an important finding.

Given the absence of a beneficial effect by adding sc-IL-2 to an $8 \mathrm{~h}$ infusion of dinutuximab beta [12], the standard treatment recommended by SIOPEN is dinutuximab beta with isotretinoin for maintenance therapy of high-risk neuroblastoma. The benefits might be less in some subgroups $(<1.5$ years, $M Y C N$-amplified localised disease) and needs close monitoring in future studies. Modifications of the length of the dinutuximab beta schedule and immunotherapy combination strategies may optimise the benefits of immunotherapy in high-risk neuroblastoma to improve survival.

Author Contributions: Conceptualization: R.L. (Ruth Ladenstein), U.P., H.N.L. Study design: R.L. (Ruth Ladenstein), U.P., H.N.L. Resources: R.L. (Ruth Ladenstein), R.L. (Roberto Luksch), V.C., S.A., G.L., P.B., J.M.M., C.O., T.T., G.C.F.C., G.S., E.R., H.S., M.B.-P., G.S., H.L., P.A., K.H., M.R.C., M.N.G., A.G. Quality control of data and algorithms: R.L. (Ruth Ladenstein), U.P. Data analysis and interpretation: R.L. (Ruth Ladenstein), U.P., H.N.L. Formal analysis: U.P. Writing-original draft preparation: R.L. (Ruth Ladenstein), U.P., A.D.J.P., H.N.L. Writing-review and editing: R.L. (Ruth Ladenstein), U.P., H.N.L., A.D.J.P., D.V.-C M.N.G. Project administration: R.L. (Ruth Ladenstein). All authors have read and agreed to the published version of the manuscript.

Funding: The funding of the European Commission 5th Frame Work Grant (SIOPEN-R-NET EC grant No. QLRI-CT-2002-01768, www.siopen-r-net.org) is disclosed as a source of funding in the author form. Pierre Fabre Médicament provided Busilvex ${ }^{\circledR}$ (Paris, France) and APEIRON (Vienna, Austria) provided dinutuximab beta (ch14.18/CHO), along with the St. Anna Kinderkrebsforschung (Vienna, Austria). The European Commission, Pierre Fabre Médicament and APEIRON had no involvement in the conduct of the research and preparation of the article. The St. Anna Kinderkrebsforschung was the academic sponsor of the trial. In addition, this work was supported by these charities, as follows (in alphabetic order): France: Fondation ARC (Association pour la Recherche sur le Cancer), Villejuif; SFCE (Société Française de Lutte contre les Cancers et Leucémies de l'Enfant et de l'Adolescent), Paris; Enfant Cancers et Santé, Montfaucon and Enfance et Cancer Association Hubert Gouin, Divonne-les-Bains. Israel: Hayim Association-for Children with Cancer in Israel, Ramat Gan. Italy: Fondazione Italiana per la Lotta al Neuroblastoma O.N.L.U.S. c/o Istituto G. Gaslini. Genova: Associazione Bianca Garavaglia O.N.L.U.S., Busto Arsizio. Switzerland: Oncosuisse, Bern; Swiss Cancer League, Bern; Fond'action contre le Cancer, Lausanne and FORCE (Fondation Recherche sur le Cancer de l'Enfant), Ecublens. UK: Cancer Research UK, London and The Neuroblastoma Society, London.

Acknowledgments: Re-cloning and production of the ch14.18 monoclonal antibody was done at Polymun, Vienna, Austria and was enabled by a SIOPEN fundraising effort in 2001. APEIRON provided additional products at a later stage. Neither Polymun nor APEIRON had a role in the study design or analysis. The authors express their gratitude and appreciation to SIOPEN investigators, treating physicians, clinical research and care teams and most importantly to patients and families facing high-risk neuroblastoma for their committed participation in the trial. The authors are indebted to Ingrid Pribill for her important technical assistance in trial management and to Mag. Claudia Zeiner-Koglin for editorial assistance.

Conflicts of Interest: The academic data supported APEIRON to obtain the dinutuximab beta product licensure in May 2017 in the European Union (EMA). SIOPEN and CCRI established a contract with APEIRON regarding the provision of academic data. Ruth Ladenstein and Holger Lode acted as consultants for APEIRON on behalf of SIOPEN for the ch14.18/CHO development. The other authors declare no conflict of interest. 


\section{References}

1. London, W.B.; Castleberry, R.P.; Matthay, K.K.; Look, A.T.; Seeger, R.C.; Shimada, H.; Thorner, P.; Brodeur, G.; Maris, J.M.; Reynolds, C.P.; et al. Evidence for an age cutoff greater than 365 days for neuroblastoma risk group stratification in the Children's Oncology Group. J. Clin. Oncol. 2005, 23, 6459-6465. [CrossRef] [PubMed]

2. Valteau-Couanet, D.; Le Deley, M.-C.; Bergeron, C.; Ducassou, S.; Michon, J.; Rubie, H.; Le Teuff, G.; Coze, C.; Plantaz, D.; Sirvent, N.; et al. Long-term results of the combination of the N7 induction chemotherapy and the busulfan-melphalan high dose chemotherapy. Pediatr. Blood Cancer 2014, 61, 977-981. [CrossRef] [PubMed]

3. Matthay, K.K.; Reynolds, C.P.; Seeger, R.C.; Shimada, H.; Adkins, E.S.; Haas-Kogan, D.; Gerbing, R.B.; London, W.B.; Villablanca, J.G. Long-term results for children with high-risk neuroblastoma treated on a randomized trial of myeloablative therapy followed by 13-cis-retinoic acid: A children's oncology group study. J. Clin. Oncol. 2009, 27, 1007-1013. [CrossRef] [PubMed]

4. Pearson, A.D.J.; Pinkerton, C.R.; Lewis, I.J.; Imeson, J.; Ellershaw, C.; Machin, D. High-dose rapid and standard induction chemotherapy for patients aged over 1 year with stage 4 neuroblastoma: A randomised trial. Lancet. Oncol. 2008, 9, 247-256. [CrossRef]

5. Ladenstein, R.; Valteau-Couanet, D.; Brock, P.; Yaniv, I.; Castel, V.; Laureys, G.; Malis, J.; Papadakis, V.; Lacerda, A.; Ruud, E.; et al. Randomized Trial of prophylactic granulocyte colony-stimulating factor during rapid COJEC induction in pediatric patients with high-risk neuroblastoma: The European HR-NBL1/SIOPEN study. J. Clin. Oncol. 2010, 28, 3516-3524. [CrossRef] [PubMed]

6. Ladenstein, R.; Pötschger, U.; Pearson, A.D.J.; Brock, P.; Luksch, R.; Castel, V.; Yaniv, I.; Papadakis, V.; Laureys, G.; Malis, J.; et al. Busulfan and melphalan versus carboplatin, etoposide, and melphalan as high-dose chemotherapy for high-risk neuroblastoma (HR-NBL1/SIOPEN): An international, randomised, multi-arm, open-label, phase 3 trial. Lancet Oncol. 2017, 18, 500-514. [CrossRef]

7. Yu, A.L.; Gilman, A.L.; Ozkaynak, M.F.; London, W.B.; Kreissman, S.G.; Chen, H.X.; Smith, M.; Anderson, B.; Villablanca, J.G.; Matthay, K.K.; et al. Anti-GD 2 antibody with GM-CSF, interleukin-2, and isotretinoin for neuroblastoma. N. Engl. J. Med. 2010, 363, 1324-1334. [CrossRef]

8. Cheung, N.-K.V.; Dyer, M.A. Neuroblastoma: Developmental biology, cancer genomics and immunotherapy. Nat. Rev. Cancer 2013, 13, 397-411. [CrossRef]

9. Zeng, Y.; Fest, S.; Kunert, R.; Katinger, H.; Pistoia, V.; Michon, J.; Lewis, G.; Ladenstein, R.; Lode, H.N. Anti-neuroblastoma effect of ch14.18 antibody produced in $\mathrm{CHO}$ cells is mediated by NK-cells in mice. Mol. Immunol. 2005, 42, 1311-1319. [CrossRef]

10. Ladenstein, R.; Weixler, S.; Baykan, B.; Bleeke, M.; Kunert, R.; Katinger, D.; Pribill, I.; Glander, P.; Bauer, S.; Pistoia, V.; et al. Ch14.18 antibody produced in $\mathrm{CHO}$ cells in relapsed or refractory Stage 4 neuroblastoma patients: A SIOPEN Phase 1 study. MAbs 2013, 5, 801-809. [CrossRef]

11. Ladenstein, R.; Pötschger, U.; Siabalis, D.; Garaventa, A.; Bergeron, C.; Lewis, I.; Stein, J.; Kohler, J.; Shaw, P.J.; Holter, W.; et al. Dose Finding Study for the Use of Subcutaneous Recombinant Interleukin-2 to Augment Natural Killer Cell Numbers in an Outpatient Setting for Stage 4 Neuroblastoma After Megatherapy and Autologous Stem-Cell Reinfusion. J. Clin. Oncol. 2011, 29, 441-448. [CrossRef] [PubMed]

12. Ladenstein, R.; Pötschger, U.; Valteau-Couanet, D.; Luksch, R.; Castel, V.; Yaniv, I.; Laureys, G.; Brock, P.; Michon, J.M.; Owens, C.; et al. Interleukin 2 with anti-GD $\mathrm{GD}_{2}$ antibody ch14.18/CHO (dinutuximab beta) in patients with high-risk neuroblastoma (HR-NBL1/SIOPEN): A multicentre, randomised, phase 3 trial. Lancet Oncol. 2018, 19, 1617-1629. [CrossRef]

13. Park, J.R.; Kreissman, S.G.; London, W.B.; Naranjo, A.; Cohn, S.L.; Hogarty, M.D.; Tenney, S.C.; Haas-Kogan, D.; Shaw, P.J.; Kraveka, J.M.; et al. Effect of Tandem Autologous Stem Cell Transplant vs Single Transplant on Event-Free Survival in Patients With High-Risk Neuroblastoma: A Randomized Clinical Trial. JAMA 2019, 322, 746-755. [CrossRef] [PubMed]

14. Mody, R.; Naranjo, A.; Van Ryn, C.; Yu, A.L.; London, W.B.; Shulkin, B.L.; Parisi, M.T.; Servaes, S.-E.-N.; Diccianni, M.B.; Sondel, P.M.; et al. Irinotecan-temozolomide with temsirolimus or dinutuximab in children with refractory or relapsed neuroblastoma (COG ANBL1221): an open-label, randomised, phase 2 trial. Lancet Oncol. 2017, 18, 946-957. [CrossRef] 
15. Furman, W.L.; Federico, S.M.; McCarville, M.B.; Shulkin, B.L.; Davidoff, A.M.; Krasin, M.J.; Sahr, N.; Sykes, A.; Wu, J.; Brennan, R.C.; et al. A Phase II Trial of Hu14.18K322A in Combination with Induction Chemotherapy in Children with Newly Diagnosed High-Risk Neuroblastoma. Clin. Cancer Res. 2019, 25, 6320-6328. [CrossRef] [PubMed]

16. Brodeur, G.M.; Pritchard, J.; Berthold, F.; Carlsen, N.L.; Castel, V.; Castelberry, R.P.; De Bernardi, B.; Evans, A.E.; Favrot, M.; Hedborg, F. Revisions of the international criteria for neuroblastoma diagnosis, staging, and response to treatment. J. Clin. Oncol. 1993, 11, 1466-1477. [CrossRef]

17. Ambros, I.M.; Benard, J.; Boavida, M.; Bown, N.; Caron, H.; Combaret, V.; Couturier, J.; Darnfors, C.; Delattre, O.; Freeman-Edward, J.; et al. Quality Assessment of Genetic Markers Used for Therapy Stratification. J. Clin. Oncol. 2003, 21, 2077-2084. [CrossRef]

18. Amoroso, L.; Erminio, G.; Makin, G.; Pearson, A.D.; Brock, P.; Valteau-Couanet, D.; Castel, V.; Pasquet, M.; Laureys, G.; Thomas, C.; et al. Topotecan-Vincristine-Doxorubicin in Stage 4 High Risk Neuroblastoma Patients Failing to Achieve a Complete Metastatic Response to Rapid COJEC-a SIOPEN Study. Cancer Res. Treat. 2018, 50, e148. [CrossRef]

19. Peto, R.; Pike, M.C.; Armitage, P.; Breslow, N.E.; Cox, D.R.; Howard, S.V.; Mantel, N.; McPherson, K.; Peto, J.; Smith, P.G. Design and analysis of randomized clinical trials requiring prolonged observation of each patient. II. analysis and examples. Br. J. Cancer 1977, 35, 1-39. [CrossRef]

20. Kalbfleisch, J.D.; Prentice, R.L. The Statistical Analysis of Failure Time Data, 2nd ed.; John Wiley \& Sons: Hoboken, NJ, USA, 2002; Chapter 8.3.3; pp. 251-254. ISBN 0-471-36357-X.

21. Gray, R.J. A Class of K-Sample Tests for Comparing the Cumulative Incidence of a Competing Risk. Ann. Stat. 1988, 16, 1141-1154. [CrossRef]

22. Andersen, P.K.; Perme, M.P. Pseudo-observations in survival analysis. Stat. Methods Med. Res. 2010, 19, 71-99. [CrossRef]

23. Simon, T.; Hero, B.; Faldum, A.; Handgretinger, R.; Schrappe, M.; Niethammer, D.; Berthold, F. Consolidation treatment with chimeric anti-GD $\mathrm{G}_{2}$-antibody ch14.18 in children older than 1 year with metastatic neuroblastoma. J. Clin. Oncol. 2004, 22, 3549-3557. [CrossRef] [PubMed]

24. Simon, T.; Hero, B.; Faldum, A.; Handgretinger, R.; Schrappe, M.; Niethammer, D.; Berthold, F. Infants with Stage 4 Neuroblastoma: The Impact of the Chimeric Anti-GD - -Antibody ch14.18 Consolidation Therapy. Klin. Pädiatrie 2005, 217, 147-152. [CrossRef] [PubMed]

25. Simon, T.; Hero, B.; Faldum, A.; Handgretinger, R.; Schrappe, M.; Klingebiel, T.; Berthold, F. Long term outcome of high-risk neuroblastoma patients after immunotherapy with antibody ch14.18 or oral metronomic chemotherapy. BMC Cancer 2011, 11, e21. [CrossRef] [PubMed] 